1 PRECIOUS: PREvention of Complications to Improve OUtcome in elderly patients with acute Stroke. Statistical analysis plan of a randomised, open, phase III, clinical trial with blinded outcome assessment

Jeroen C de Jonge, ${ }^{1} \dagger$ Lisa J Woodhouse, ${ }^{2} \dagger$ Hendrik Reinink, ${ }^{1} \dagger$ H Bart van der Worp, ${ }^{1} \uparrow$ Philip

7

$8{ }^{1}$ Department of Neurology and Neurosurgery, Brain Center, University Medical Center 9 Utrecht, Utrecht University, Utrecht, The Netherlands.

${ }^{2}$ Stroke Trials Unit, Division of Clinical Neuroscience, University of Nottingham,

11 Nottingham, United Kingdom

${ }^{3}$ Stroke, Nottingham University Hospitals NHS Trust, Nottingham, United Kingdom

$\dagger+$ These authors contributed equally to this paper.

Chief investigator / corresponding author:

H. Bart van der Worp, MD, PhD

Department of Neurology and Neurosurgery

Brain Center, University Medical Center Utrecht, Utrecht University

Heidelberglaan 1003584 CX Utrecht, The Netherlands

E: $\underline{\text { H.B.vanderWorp@umcutrecht.nl }}$

Statistician: L.J. Woodhouse

Trial registration number: ISRCTN82217627

Key words: stroke, complications, elderly, ceftriaxone, metoclopramide, paracetamol 
ABSTRACT

30

Rationale. Aspiration, infections, and fever are common in the first days after stroke, especially in older patients. The occurrence of these complications has been associated with an increased risk of death or dependency.

Aims and design. PREvention of Complications to Improve OUtcome in elderly patients with acute Stroke (PRECIOUS) is an international, multi-centre, $3 \times 2$ factorial, randomised, controlled, open-label clinical trial with blinded outcome assessment, which will assess whether prevention of aspiration, infections, or fever with metoclopramide, ceftriaxone, paracetamol, respectively, or any combination of these, in the first four days after stroke onset improves functional outcome at 90 days in elderly patients with acute stroke.

Discussion. This statistical analysis plan provides a technical description of the statistical methodology and unpopulated tables and figures. The paper is written prior to data lock and unblinding of treatment allocation.

45

47 prospectively registered). 
In the first days after stroke, about half of all patients develop one or more complications, including aspiration, infections, or fever. The risk of developing these events is greater in patients of higher age or with more severe stroke (1-3). These complications can impede functional recovery, prolong hospital admissions, and are independently associated with an increased risk of death or long-term dependency $(1,2,4-11)$. The risk of developing these complications can be reduced by very simple, safe and inexpensive measures, such as metoclopramide for the management of dysphagia, antibiotics for the prevention of infections, and paracetamol for the prevention of fever, but it is uncertain whether these measures also improve functional outcome (12-15). In some, generally small, randomised trials, preventive treatment with these drugs not only convincingly reduced the risks of aspiration, infections, or fever by one third to one half, but was also associated with clear trends towards a lower risk of death or poor outcome (12-15). However, in two large randomised clinical trials, preventive treatment with antibiotics did not improve functional outcomes $(16,17)$. Guidelines of the European Stroke Organisation concluded that there is insufficient evidence from randomised trials to make strong recommendations on whether, when and to whom preventive antibiotic or antipyretic treatment should be given after ischaemic stroke or intracerebral haemorrhage $(18,19)$. The PREvention of Complications to Improve OUtcome in elderly patients with acute Stroke (PRECIOUS) trial will assess whether prevention of aspiration, infections, or fever with metoclopramide, ceftriaxone, paracetamol, or any combination of these in the first four days after stroke onset improves functional outcome at 90 days in older patients with acute stroke. The current paper describes the statistical analysis plan (SAP) of the trial and conforms to the guidelines set by Gamble et al (20). The details of the study protocol of the PRECIOUS trial have been published earlier 
(21). PRECIOUS has received funding from the European Union's Horizon 2020 research and innovation programme under grant agreement No 634809.

\section{Study methods}

PRECIOUS is an international, multi-centre, multi-factorial, randomised, controlled, phaseIII, open-label clinical trial with blinded outcome assessment (PROBE). The primary objective is to assess whether prevention of aspiration, infections, or fever with metoclopramide, ceftriaxone, paracetamol, or any combination of these in the first four days after stroke onset improves functional outcome at 90 days in older patients with acute stroke. Patients will be randomly allocated in a $2 * 2 * 2$ factorial design to any combination of openlabel oral, rectal, or intravenous metoclopramide (10 mg thrice daily); intravenous ceftriaxone (2000 mg once daily); oral, rectal, or intravenous paracetamol (1000 mg four times daily), or usual care, started within 24 hours after symptom onset and continued for 4 days or until complete recovery or discharge from hospital, if earlier. In patients with moderate to severe renal impairment or with severe hepatic impairment, the dose of metoclopramide is reduced to $5 \mathrm{mg}$ thrice daily, and in patients with end-stage renal disease to $2.5 \mathrm{mg}$ thrice daily. Patients will be stratified according to country (Estonia, Germany, Greece, Hungary, Italy, the Netherlands, Norway, Poland, United Kingdom) and there will be 5 minimisation factors: age (66 - 75 years; > 75 years), sex (male vs. female), stroke type (ischaemic stroke vs. intracerebral haemorrhage), stroke severity (NIHSS 6-12 vs. >12) and diabetes Mellitus (yes vs. no). 3800 patients will be recruited, based on the sample size calculation described in the previously published protocol (21).

\section{Statistical interim analyses and stopping guidance}


An independent Data and Safety Monitoring Board (DSMB) will conduct unblinded interim analyses after $600,1200,1800,2400$, and 3000 patients have completed follow-up to assess the safety of the interventions in the trial. With respect to efficacy, the DSMB will conduct unblinded interim analyses after 2400 patients had their final follow-up. DSMB members will receive listings of all SAE reports as well as unblinded aggregate summaries of data by treatment groups for review in closed meetings. The results of these interim analyses are confidential and limited to the members of DSMB.

\section{Timing of final analysis}

This statistical analysis plan (SAP) will be signed off by the trial Steering Committee and then submitted for publication prior to data lock and final analysis. The final statistical analysis will be performed once recruitment has ceased, final follow-up and final outcome adjudication have been completed, final data have been checked and any errors corrected, and the database has been locked. The analyses will be carried out according to the current statistical analysis plan. The statistical analyses will be performed by the Nottingham Stroke Trial Unit (NSTU) at the University of Nottingham (UNOTT) in collaboration with the UMC Utrecht.

\section{Trial population}

The study population will consist of patients aged 66 years or older who are hospitalised with moderately severe to severe (National Institutes of Health Stroke Scale (NIHSS) $\geq 6$ ) acute ischaemic stroke or intracerebral haemorrhage. Patients will only be included if treatment can be started within 24 hours of stroke onset. For a complete overview of the inclusion and exclusion criteria, we refer to the study protocol (21). Patients are planned to be recruited in 
about 80 hospitals in 9 European countries over a period of about four years. To increase the generalisability of the findings, these countries are distributed across Europe, and include Estonia, Germany, Greece, Hungary, Italy, the Netherlands, Norway, Poland, and the United Kingdom. For the same reason, the trial will recruit patients both in academic and regional hospitals.

\section{Statistical Analysis}

Primary outcome

The primary outcome measure is the score on the modified Rankin Scale (mRS) at 90 days $( \pm$ 14 days). The $\mathrm{mRS}$ is an ordinal scale ranging from 0-6 (22). The mRS assessment at 90 days will be during a hospital/home visit or by telephone, and the assessment or a report thereof will be recorded using a digital video camera. Three blinded raters will view the videotape and adjudicate a score on the mRS.

\section{Primary outcome analysis}

For each patient, a median mRS score will be calculated from the three mRS scores obtained through centralised adjudications by raters who are blinded to treatment allocation. The use of three scores increases the precision in scoring and statistical power as compared to a single $\mathrm{mRS}$ assessment (23). The primary effect estimate will be the difference in the mRS scores between the active treatment group and controls assessed using ordinal logistic regression, and will be expressed as an odds ratio with $95 \%$ confidence interval (24). The primary analysis will be performed on all randomised patients with a valid mRS score at 90 days. The distribution of the mRS scores will be shown as a figure. Three separate primary analyses will be performed for each intervention versus their respective controls (e.g. metoclopramide vs. non-metoclopramide). The primary analyses will be adjusted for stratification (country), 
minimisation (age, sex, stroke type, stroke severity, diabetes), and other baseline prognostic thrombectomy], time from onset to randomisation) factors, and treatment allocation for the other two strata of the trial.

Primary outcome subgroup analysis

Comparison of the effect of the three intervention groups vs. their respective controls on the primary outcome will be performed in the following pre-specified subgroups (assuming sufficient numbers in each subgroup) with assessment of interaction between treatment and the minimisation factors (these subgroup analyses are considered hypothesis-generating):

- $\quad$ Age $(\leq 75,>75$ years $)$;

- Sex (male, female);

- Stroke type (ischaemic stroke, intracerebral haemorrhage);

In addition, the interaction between treatment and other baseline factors will be assessed:

- Presence of atrial fibrillation (yes, no);

- $\quad$ Pre-stroke mRS score $(0,>0)$;

- Reperfusion treatment (alteplase and/or mechanical thrombectomy);

- $\quad$ Time to treatment ( $<6, \geq 6$ hours $<12$ hours, $\geq 12$ hours);

- Treatment allocation for the other two trial strata (paracetamol - active, control; ceftriaxone - active, control; metoclopramide - active, control). Since the study is not powered to detect interactions between the three interventions, these interactions will be investigated in secondary analyses. 
Sensitivity analyses

172 Four sensitivity analyses of the mRS will also be performed: unadjusted ordinal logistic

173 regression; adjusted analysis of mRS following regression imputation of missing data;

174 multiple linear regression on the mean mRS score for each participant, and binary logistic

175 regression on $\mathrm{mRS}>2$.

Secondary outcomes

The following secondary outcomes will be assessed at 7 days ( \pm 1 day) or at discharge, if earlier:

- Infections in the first 7 days ( \pm 1 day; frequency, type, and Clostridium difficile

The following secondary outcomes will be assessed at 90 days ( \pm 14 days):

- Death; 
- Unfavourable functional outcome, defined as mRS 3 to 6 ;

- Disability assessed with the score on the Barthel Index (BI);

- Cognition assessed with the Montreal Cognitive Assessment (MoCA);

- Quality of life assessed with the EuroQol 5D-5L (EQ-5D-5L), and EQ-visual analogue scale (EQ-VAS)

- Home time: the number of nights among the first 90 since stroke onset that are spent in the patient's own home or a relative's home. Resource use will be censored at 90 days. Where final follow-up occurs earlier, the last known placement will be extrapolated to 90 days;

- Patient location over first 90 days ( \pm 14 days): hospital; rehabilitation service; chronic nursing facility; home.

\section{Analysis of secondary outcomes}

Binary logistic regression will be used for binary outcomes (e.g. $m R S>2$ ). Cox proportional hazards regression for time to events (e.g. death). Ordinal logistic regression will be used for ordered categorical data (e.g. mRS). Multiple linear regression will be used for continuous outcomes (e.g. BI, EQ-VAS). Patients with missing outcome data will be excluded from the analysis.

\section{Missing data and death}

Patients without a primary outcome assessment at $90 \pm 14$ days will be considered as a lost to follow-up. The total amount of patients who are lost to follow-up will be recorded and calculated for each treatment arm. The primary analysis will be performed on all randomised patients with a valid mRS score at 90 days. In a sensitivity analysis, missing mRS data will be imputed using multiple regression-based imputation. 
221 For the secondary outcome measures (Barthel Index, MoCA, EQ-5D-5L, EQ-VAS), patients

222 who die will be assigned a value one unit worse than any living value. This way, patients who 223 die cannot be given a score similar to the worst score of patients who are alive, and it ensures 224 that all patients will be included in the analysis. Potential scores, with worst with dead added, 225 are:

- Modified Rankin Scale (mRS), 0 to 5 with death $=6$

227 - Barthel Index (BI), 100 to 0 with death $=-5$

- EuroQol 5D-5L (EQ-5D-5L), -0.5 to 1 with death $=0$

- EuroQol visual analogue scale (EQ-VAS), 0 to 100 with death $=-1$

- Montreal cognitive assessment (MoCA), 0 to 30 with death $=-1$.

\section{Safety outcomes}

233

In the first 7 days after randomisation, all SAEs will be reported and described by duration (start and stop dates), severity, outcome, treatment, and relation to the investigational medical product (IMP), or if unrelated, the cause. All SAEs will be tabulated per treatment stratum. In addition, any SAE occurring between day 7 and the end of follow-up on day 90 ( \pm 14 days) for which a causal relationship between the IMP and the SAE is considered at least a reasonable possibility (i.e., SARs and SUSARs) should be reported as other SAEs.

\section{Treatment restrictions}

The presence of any treatment restriction will be recorded at baseline and during the hospital phase, and classified as 1 . Do not resuscitate; 2. Do not intubate and ventilate; 3 . Withhold other treatments that may prolong life; 4. Withhold food; 5. Withhold fluids; and 6. Palliation (e.g. with morphine or a benzodiazepine). Any combination of these strategies is possible. 
The primary study will report on the frequency of each treatment restriction, further analyses on this topic will be published in future sub-group analyses.

\section{Minimising bias}

PRECIOUS is an open-label clinical trial and both patients and treating physicians are therefore aware of the assigned treatment. Knowledge of treatment allocation can influence outcome assessment, and unblinded trials like PRECIOUS are therefore at risk of detection bias. In addition, despite its apparent simplicity, assessment of the score on the mRS has been associated with considerable inter-observer variability, especially in multicentre studies, and may therefore affect trial power and treatment effect size. In PRECIOUS, these two major issues are minimised through 1) online training and certification of outcome assessors via a link on the PRECIOUS website; and 2) central outcome assessment by three blinded adjudicators based on digital video recordings of the 90-day outcome interviews. This central adjudication by trained adjudicators offers several benefits (23):

1. Blinding is assured;

2. Standardisation is possible across multiple regions and cultures;

3. Statistical power is enhanced through the use of three repeated assessments;

4. The estimate of treatment effect size is restored (since statistical noise leads to underestimation)

5. It provides independent validation of the information that is collected, thereby minimising the risk of fraud;

6. Site staff perform to a higher standard when aware that there will be review or audit of their activity. 
In addition, the risk of bias is reduced by performing the statistical analyses according to the intention-to-treat principle and adjusting for the minimisation factors, other relevant baseline characteristics, and treatment allocation for the other two strata of the trial.

\section{Statistical principles}

274 Confidence intervals and P values

275 Analyses will be two-sided $\mathrm{p}<0.05$ with $95 \%$ confidence intervals presented. The trial is testing the effect of the interventions on $\mathrm{mRS}$ and analyses in subgroups and on other outcomes are considered hypothesis-generating. Hence, no adjustment will be made for multiplicity of testing.

\section{Alpha spending}

The Data Monitoring Committee performs safety assessments using the Haybittle-Peto boundary rule $(\mathrm{p}<0.001)$; hence, no significant spending of alpha will occur during the trial. All analyses will be two-tailed and p-values of $<0.05$ will denote statistical significance; $95 \%$ confidence intervals will be provided. Adjustment for multiple comparisons will not be performed but all contrasts will be declared.

\section{Compliance}

288 Compliance with allocated treatment will be tabulated. For each of the three study drugs, the number of received dosages will be calculated (maximum of four for ceftriaxone, twelve for metoclopramide and sixteen for paracetamol). The number of patients who received the first dosage within the time window of 24 hours will also be presented; if the dosage was not given within 24 hours, the reason will be given (withdrawn informed consent, death, human error, other reason). 
All efficacy analyses will be performed on the intention-to-treat population. The robustness of the primary and key secondary analyses will be assessed in the per-protocol population. Safety analyses will be performed on the safety population.

The following population definitions will be used: used.

Patients with protocol violations in trial eligibility will be included in the intention to treat population, but excluded in the per-protocol analysis. Patients who withdrew informed consent before initiating treatment will be excluded from analysis. If (per accident) multiple randomisations are performed for a single patient, the result of the first randomisation will be

- Intention-to-treat in primary efficacy analysis: All randomised participants who received any study medication and with a valid mRS score recorded at 90 days.

- Intention-to-treat in primary safety analysis: All randomised participants with a vital status recorded at 90 days.

- Per-protocol: All participants in the intention-to-treat population who are deemed to have no major protocol violations that could interfere with the objectives of the study.

\section{Current status}

The trial received approval from the central Medical Ethics Committee of the University Medical Center Utrecht, The Netherlands on 3 February 2016. The Dutch National Competent Authority (Centrale Commissie Mensgebonden Onderzoek (CCMO)), declared to have no objection against the execution of the clinical trial within the Netherlands on 17 
319 November 2015. In addition, the national (and local, if applicable) medical ethical

320 committees and competent authorities of the other 8 participating countries have approved

321 the trial. The first patient was included in May 2016. The analysis and reporting of the trial

322 will be in accordance with CONSORT guidelines. After publication of the trial, to promote

323 the independent re-use of PRECIOUS data, a coded dataset will be made available in a public

324 data repository within 18 months of the final follow-up of the last patient. Coded data will

325 also be included in the Virtual International Stroke Trials Archive (VISTA). 
Table 1. Baseline characteristics

\begin{tabular}{|c|c|c|c|c|c|c|c|}
\hline & All & Paracetamol & Control & Metoclopramide & Control & Ceftriaxone & Control \\
\hline \multicolumn{8}{|l|}{ Total patients randomised } \\
\hline Age (years) & Mean (SD) & Mean (SD) & Mean (SD) & Mean (SD) & Mean (SD) & Mean (SD) & Mean (SD) \\
\hline Sex, male $(\%)$ & $\mathrm{n}(\%)$ & $\mathrm{n}(\%)$ & $\mathrm{n}(\%)$ & $\mathrm{n}(\%)$ & $\mathrm{n}(\%)$ & $\mathrm{n}(\%)$ & $\mathrm{n}(\%)$ \\
\hline Premorbid mRS [/6] & Median & Median & Median & Median & Median & Median & Median \\
\hline & [IQR] & [IQR] & [IQR] & [IQR] & [IQR] & [IQR] & [IQR] \\
\hline Ethnicity, white (\%) & $\mathrm{n}(\%)$ & $\mathrm{n}(\%)$ & $\mathrm{n}(\%)$ & $\mathrm{n}(\%)$ & $\mathrm{n}(\%)$ & $\mathrm{n}(\%)$ & $\mathrm{n}(\%)$ \\
\hline \multicolumn{8}{|l|}{ Medical History (\%) } \\
\hline - Atrial fibrillation & $\mathrm{n}(\%)$ & $\mathrm{n}(\%)$ & $\mathrm{n}(\%)$ & $\mathrm{n}(\%)$ & $\mathrm{n}(\%)$ & $\mathrm{n}(\%)$ & $\mathrm{n}(\%)$ \\
\hline - Hypercholesterolaemia & $\mathrm{n}(\%)$ & $\mathrm{n}(\%)$ & $\mathrm{n}(\%)$ & $\mathrm{n}(\%)$ & $\mathrm{n}(\%)$ & $\mathrm{n}(\%)$ & $\mathrm{n}(\%)$ \\
\hline - Hypertension & $\mathrm{n}(\%)$ & $\mathrm{n}(\%)$ & $\mathrm{n}(\%)$ & $\mathrm{n}(\%)$ & $\mathrm{n}(\%)$ & $\mathrm{n}(\%)$ & $\mathrm{n}(\%)$ \\
\hline - Diabetes mellitus & $\mathrm{n}(\%)$ & $\mathrm{n}(\%)$ & $\mathrm{n}(\%)$ & $\mathrm{n}(\%)$ & $\mathrm{n}(\%)$ & $\mathrm{n}(\%)$ & $\mathrm{n}(\%)$ \\
\hline $\begin{array}{l}\text { - Obstructive pulmonary } \\
\text { disease }\end{array}$ & $\mathrm{n}(\%)$ & $\mathrm{n}(\%)$ & $\mathrm{n}(\%)$ & $\mathrm{n}(\%)$ & $\mathrm{n}(\%)$ & $\mathrm{n}(\%)$ & $\mathrm{n}(\%)$ \\
\hline - Previous stroke & $\mathrm{n}(\%)$ & $\mathrm{n}(\%)$ & $\mathrm{n}(\%)$ & $\mathrm{n}(\%)$ & $\mathrm{n}(\%)$ & $\mathrm{n}(\%)$ & $\mathrm{n}(\%)$ \\
\hline - Immunocompromised & $\mathrm{n}(\%)$ & $\mathrm{n}(\%)$ & $\mathrm{n}(\%)$ & $\mathrm{n}(\%)$ & $\mathrm{n}(\%)$ & $\mathrm{n}(\%)$ & $\mathrm{n}(\%)$ \\
\hline Smoking, current & & & & & & & \\
\hline - Never & $\mathrm{n}(\%)$ & $\mathrm{n}(\%)$ & $\mathrm{n}(\%)$ & $\mathrm{n}(\%)$ & $\mathrm{n}(\%)$ & $\mathrm{n}(\%)$ & $\mathrm{n}(\%)$ \\
\hline - Ever & $\mathrm{n}(\%)$ & $\mathrm{n}(\%)$ & $\mathrm{n}(\%)$ & $\mathrm{n}(\%)$ & $\mathrm{n}(\%)$ & $\mathrm{n}(\%)$ & $\mathrm{n}(\%)$ \\
\hline - Currently & $\mathrm{n}(\%)$ & $\mathrm{n}(\%)$ & $\mathrm{n}(\%)$ & $\mathrm{n}(\%)$ & $\mathrm{n}(\%)$ & $\mathrm{n}(\%)$ & $\mathrm{n}(\%)$ \\
\hline \multicolumn{8}{|l|}{$\begin{array}{l}\text { Pre-stroke method of food } \\
\text { intake }\end{array}$} \\
\hline - Normal food & $\mathrm{n}(\%)$ & $\mathrm{n}(\%)$ & $\mathrm{n}(\%)$ & $\mathrm{n}(\%)$ & $\mathrm{n}(\%)$ & $\mathrm{n}(\%)$ & $\mathrm{n}(\%)$ \\
\hline $\begin{array}{l}\text { - Oral softened food or fluids } \\
\text { only }\end{array}$ & $\mathrm{n}(\%)$ & $\mathrm{n}(\%)$ & n $(\%)$ & $\mathrm{n}(\%)$ & $\mathrm{n}(\%)$ & $\mathrm{n}(\%)$ & $\mathrm{n}(\%)$ \\
\hline - Nasogastric tube & $\mathrm{n}(\%)$ & $\mathrm{n}(\%)$ & $\mathrm{n}(\%)$ & $\mathrm{n}(\%)$ & $\mathrm{n}(\%)$ & $\mathrm{n}(\%)$ & $\mathrm{n}(\%)$ \\
\hline $\begin{array}{l}\text { - Percutaneous endoscopic } \\
\text { gastrostomy }\end{array}$ & $\mathrm{n}(\%)$ & $\mathrm{n}(\%)$ & $\mathrm{n}(\%)$ & $\mathrm{n}(\%)$ & $\mathrm{n}(\%)$ & $\mathrm{n}(\%)$ & $\mathrm{n}(\%)$ \\
\hline - Intravenous only & $\mathrm{n}(\%)$ & $\mathrm{n}(\%)$ & $\mathrm{n}(\%)$ & $\mathrm{n}(\%)$ & $\mathrm{n}(\%)$ & $\mathrm{n}(\%)$ & $\mathrm{n}(\%)$ \\
\hline
\end{tabular}




\begin{tabular}{|c|c|c|c|c|c|c|c|}
\hline $\begin{array}{l}\text { Use of drugs } 3 \text { days before } \\
\text { randomisation } \\
\text { - Paracetamol }\end{array}$ & $\mathrm{n}(\%)$ & $\mathrm{n}(\%)$ & $\mathrm{n}(\%)$ & $\mathrm{n}(\%)$ & $\mathrm{n}(\%)$ & $\mathrm{n}(\%)$ & $\mathrm{n}(\%)$ \\
\hline - Metoclopramide & $\mathrm{n}(\%)$ & $\mathrm{n}(\%)$ & $\mathrm{n}(\%)$ & $\mathrm{n}(\%)$ & $\mathrm{n}(\%)$ & $\mathrm{n}(\%)$ & $\mathrm{n}(\%)$ \\
\hline - Ceftriaxone & $\mathrm{n}(\%)$ & $\mathrm{n}(\%)$ & $\mathrm{n}(\%)$ & $\mathrm{n}(\%)$ & $\mathrm{n}(\%)$ & $\mathrm{n}(\%)$ & $\mathrm{n}(\%)$ \\
\hline $\begin{array}{l}\text { Time, onset to randomisation } \\
\text { (min) } \\
\text { Stroke type }(\%)\end{array}$ & Mean (SD) & Mean (SD) & Mean (SD) & Mean (SD) & Mean (SD) & Mean (SD) & Mean (SD) \\
\hline Ischaemic stroke & $\mathrm{n}(\%)$ & $\mathrm{n}(\%)$ & $\mathrm{n}(\%)$ & $\mathrm{n}(\%)$ & $\mathrm{n}(\%)$ & $\mathrm{n}(\%)$ & $\mathrm{n}(\%)$ \\
\hline Intracerebral haemorrhage & $\mathrm{n}(\%)$ & $\mathrm{n}(\%)$ & $\mathrm{n}(\%)$ & $\mathrm{n}(\%)$ & $\mathrm{n}(\%)$ & $\mathrm{n}(\%)$ & $\mathrm{n}(\%)$ \\
\hline Other diagnosis & $\mathrm{n}(\%)$ & $\mathrm{n}(\%)$ & $\mathrm{n}(\%)$ & $\mathrm{n}(\%)$ & $\mathrm{n}(\%)$ & $\mathrm{n}(\%)$ & $\mathrm{n}(\%)$ \\
\hline NIHSS (/42) & Mean (SD) & Mean (SD) & Mean (SD) & Mean (SD) & Mean (SD) & Mean (SD) & Mean (SD) \\
\hline Systolic BP (mmHg) & Mean (SD) & Mean (SD) & Mean (SD) & Mean (SD) & Mean (SD) & Mean (SD) & Mean (SD) \\
\hline Diastolic BP (mmHg) & Mean (SD) & Mean (SD) & Mean (SD) & Mean (SD) & Mean (SD) & Mean (SD) & Mean (SD) \\
\hline Heart rate (bpm) & Mean (SD) & Mean (SD) & Mean (SD) & Mean (SD) & Mean (SD) & Mean (SD) & Mean (SD) \\
\hline $\begin{array}{l}\text { Body temperature }\left({ }^{\circ} \mathrm{C}\right) \\
\text { Acute stroke treatment }(\%)\end{array}$ & Mean (SD) & Mean (SD) & Mean (SD) & Mean (SD) & Mean (SD) & Mean (SD) & Mean (SD) \\
\hline - Intravenous thrombolysis & $\mathrm{n}(\%)$ & $\mathrm{n}(\%)$ & $\mathrm{n}(\%)$ & $\mathrm{n}(\%)$ & $\mathrm{n}(\%)$ & $\mathrm{n}(\%)$ & $\mathrm{n}(\%)$ \\
\hline - Mechanical thrombectomy & $\mathrm{n}(\%)$ & $\mathrm{n}(\%)$ & $\mathrm{n}(\%)$ & $\mathrm{n}(\%)$ & $\mathrm{n}(\%)$ & $\mathrm{n}(\%)$ & $\mathrm{n}(\%)$ \\
\hline
\end{tabular}

Data are n (\%) or median [IQR]. mRS, modified Rankin Scale; NIHSS, National Institutes of Health Stroke scale; BP, blood pressure. 
Table 2. Primary outcome. Analyses are adjusted except where stated

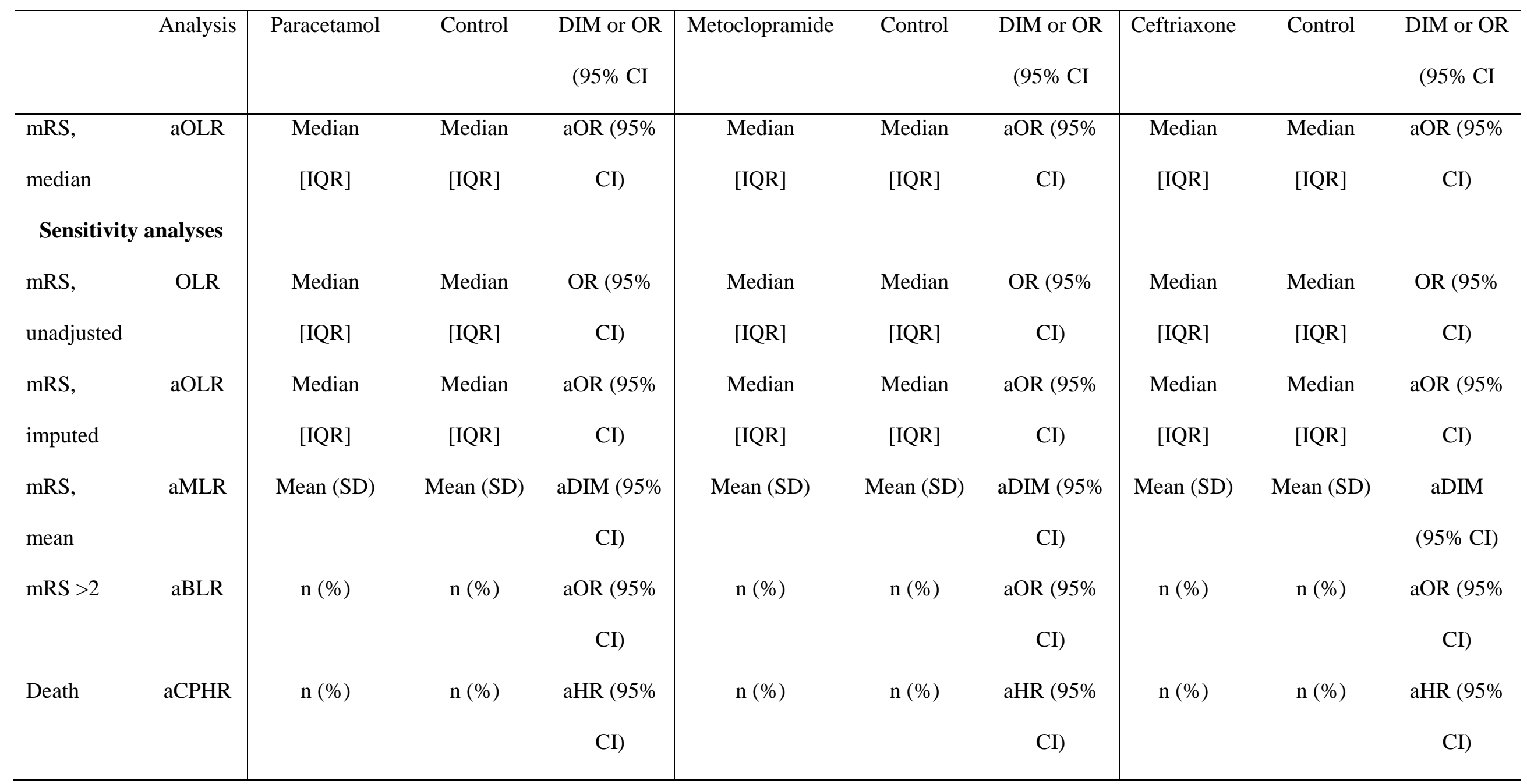

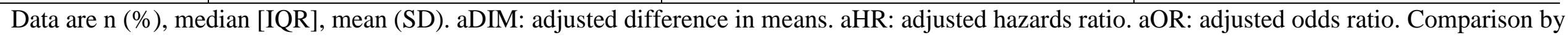
adjusted ordinal logistic regression (aOLR), multiple linear regression (aMLR), Cox proportional hazards regression (CPHR) or adjusted binary logistic regression (aBLR) 
Table 3. Secondary outcome assessment at 90 days

\begin{tabular}{|c|c|c|c|c|c|c|c|c|c|c|}
\hline & Analysis & Paracetamol & Control & $\begin{array}{l}\text { OR }(95 \% \\
\text { CI) }\end{array}$ & Metoclopramide & Control & $\begin{array}{l}\text { OR }(95 \% \\
\text { CI) }\end{array}$ & Ceftriaxone & Control & $\begin{array}{l}\text { OR }(95 \% \\
\text { CI) }\end{array}$ \\
\hline \multicolumn{11}{|l|}{ mRS, median } \\
\hline Ischaemic stroke & aOLR & $\begin{array}{l}\text { Median } \\
\text { [IQR] }\end{array}$ & $\begin{array}{l}\text { Median } \\
{[\mathrm{IQR}]}\end{array}$ & $\begin{array}{l}\mathrm{aOR}(95 \% \\
\mathrm{CI})\end{array}$ & $\begin{array}{l}\text { Median } \\
{[\mathrm{IQR}]}\end{array}$ & $\begin{array}{l}\text { Median } \\
\text { [IQR] }\end{array}$ & $\begin{array}{l}\mathrm{aOR}(95 \% \\
\mathrm{CI})\end{array}$ & $\begin{array}{l}\text { Median } \\
\text { [IQR] }\end{array}$ & $\begin{array}{l}\text { Median } \\
\text { [IQR] }\end{array}$ & $\begin{array}{l}\mathrm{aOR}(95 \% \\
\mathrm{CI})\end{array}$ \\
\hline $\begin{array}{l}\text { Intracerebral } \\
\text { haemorrhage }\end{array}$ & aOLR & $\begin{array}{l}\text { Median } \\
{[\mathrm{IQR}]}\end{array}$ & $\begin{array}{l}\text { Median } \\
{[\mathrm{IQR}]}\end{array}$ & $\begin{array}{l}\mathrm{aOR}(95 \% \\
\mathrm{CI})\end{array}$ & $\begin{array}{l}\text { Median } \\
{[\mathrm{IQR}]}\end{array}$ & $\begin{array}{l}\text { Median } \\
\text { [IQR] }\end{array}$ & $\begin{array}{l}\mathrm{aOR}(95 \% \\
\mathrm{CI})\end{array}$ & $\begin{array}{l}\text { Median } \\
\text { [IQR] }\end{array}$ & $\begin{array}{l}\text { Median } \\
{[\mathrm{IQR}]}\end{array}$ & $\begin{array}{l}\mathrm{aOR}(95 \% \\
\mathrm{CI})\end{array}$ \\
\hline Other diagnosis & $\mathrm{aOLR}$ & $\begin{array}{l}\text { Median } \\
\text { [IQR] }\end{array}$ & $\begin{array}{l}\text { Median } \\
\text { [IQR] }\end{array}$ & $\begin{array}{l}\mathrm{aOR}(95 \% \\
\mathrm{CI})\end{array}$ & $\begin{array}{l}\text { Median } \\
\text { [IQR] }\end{array}$ & $\begin{array}{l}\text { Median } \\
\text { [IQR] }\end{array}$ & $\begin{array}{l}\mathrm{aOR}(95 \% \\
\mathrm{CI})\end{array}$ & $\begin{array}{l}\text { Median } \\
\text { [IQR] }\end{array}$ & $\begin{array}{l}\text { Median } \\
\text { [IQR] }\end{array}$ & $\begin{array}{l}\mathrm{aOR}(95 \% \\
\mathrm{CI})\end{array}$ \\
\hline Mortality & aCPHR & $\mathrm{n}(\%)$ & $\mathrm{n}(\%)$ & $\begin{array}{l}\text { aHR }(95 \% \\
\text { CI) }\end{array}$ & $\mathrm{n}(\%)$ & $\mathrm{n}(\%)$ & $\begin{array}{l}\text { aHR }(95 \% \\
\text { CI) }\end{array}$ & $\mathrm{n}(\%)$ & $\mathrm{n}(\%)$ & $\begin{array}{l}\text { aHR }(95 \% \\
\mathrm{CI})\end{array}$ \\
\hline Patient location & aOLR & & & $\begin{array}{l}\mathrm{aOR}(95 \% \\
\mathrm{CI})\end{array}$ & & & $\begin{array}{l}\mathrm{aOR}(95 \% \\
\mathrm{CI})\end{array}$ & & & $\begin{array}{l}\mathrm{aOR}(95 \% \\
\mathrm{CI})\end{array}$ \\
\hline Hospital & & $\mathrm{n}(\%)$ & $\mathrm{n}(\%)$ & & $\mathrm{n}(\%)$ & $\mathrm{n}(\%)$ & & $\mathrm{n}(\%)$ & $\mathrm{n}(\%)$ & \\
\hline Rehabilitation service & & $\mathrm{n}(\%)$ & $\mathrm{n}(\%)$ & & $\mathrm{n}(\%)$ & $\mathrm{n}(\%)$ & & $\mathrm{n}(\%)$ & $\mathrm{n}(\%)$ & \\
\hline Nursing home & & $\mathrm{n}(\%)$ & $\mathrm{n}(\%)$ & & $\mathrm{n}(\%)$ & $\mathrm{n}(\%)$ & & $\mathrm{n}(\%)$ & $\mathrm{n}(\%)$ & \\
\hline Home & & $\mathrm{n}(\%)$ & $\mathrm{n}(\%)$ & & $\mathrm{n}(\%)$ & $\mathrm{n}(\%)$ & & $\mathrm{n}(\%)$ & $\mathrm{n}(\%)$ & \\
\hline $\begin{array}{l}\text { Home time (No of } \\
\text { days) }\end{array}$ & aMLR & Mean (SD) & Mean (SD) & $\begin{array}{l}\mathrm{aDIM}(95 \% \\
\mathrm{CI})\end{array}$ & Mean (SD) & Mean (SD) & $\begin{array}{l}\operatorname{aDIM}(95 \% \\
\mathrm{CI})\end{array}$ & Mean (SD) & Mean (SD) & $\begin{array}{l}\mathrm{aDIM}(95 \% \\
\mathrm{CI})\end{array}$ \\
\hline \multicolumn{11}{|l|}{ Questionnaires } \\
\hline Barthel Index & aMLR & Mean (SD) & Mean (SD) & $\begin{array}{l}\mathrm{aDIM}(95 \% \\
\mathrm{CI})\end{array}$ & Mean (SD) & Mean (SD) & $\begin{array}{l}\mathrm{aDIM}(95 \% \\
\mathrm{CI})\end{array}$ & Mean (SD) & Mean (SD) & $\begin{array}{l}\mathrm{aDIM}(95 \% \\
\mathrm{CI})\end{array}$ \\
\hline
\end{tabular}




\begin{tabular}{|c|c|c|c|c|c|c|c|c|c|c|}
\hline $\mathrm{MoCA}$ & aMLR & Mean (SD) & Mean (SD) & $\begin{array}{l}\mathrm{aDIM}(95 \% \\
\mathrm{CI})\end{array}$ & Mean (SD) & Mean (SD) & $\begin{array}{l}\text { aDIM }(95 \% \\
\text { CI })\end{array}$ & Mean (SD) & Mean (SD) & $\begin{array}{l}\mathrm{aDIM}(95 \% \\
\mathrm{CI})\end{array}$ \\
\hline EQ-5D-5L & aMLR & Mean (SD) & Mean (SD) & $\begin{array}{l}\mathrm{aDIM}(95 \% \\
\mathrm{CI})\end{array}$ & Mean (SD) & Mean (SD) & $\begin{array}{l}\text { aDIM }(95 \% \\
\text { CI })\end{array}$ & Mean (SD) & Mean (SD) & $\begin{array}{l}\text { aDIM }(95 \% \\
\text { CI) }\end{array}$ \\
\hline EQ-VAS & aMLR & Mean (SD) & Mean (SD) & $\begin{array}{l}\mathrm{aDIM}(95 \% \\
\mathrm{CI})\end{array}$ & Mean (SD) & Mean (SD) & $\begin{array}{l}\mathrm{aDIM}(95 \% \\
\mathrm{CI})\end{array}$ & Mean (SD) & Mean (SD) & $\begin{array}{l}\mathrm{aDIM}(95 \% \\
\mathrm{CI})\end{array}$ \\
\hline
\end{tabular}

Data are $\mathrm{n}(\%)$ or median [IQR]. aDIM: adjusted difference in means. aOR: adjusted odds ratio. aHR adjusted hazards ratio. Comparison by adjusted ordinal logistic regression (aOLR), Cox Proportional Hazards regression (aCPHR) or multiple linear regression (aMLR). mRS, modified Rankin Scale; MoCA, Montreal Cognitive Assessment; EQ-5D-5L, EuroQol 5D-5L; EQ-VAS, EuroQol-Visual Analogue Scale 


\section{Figure 1. Trial profile}

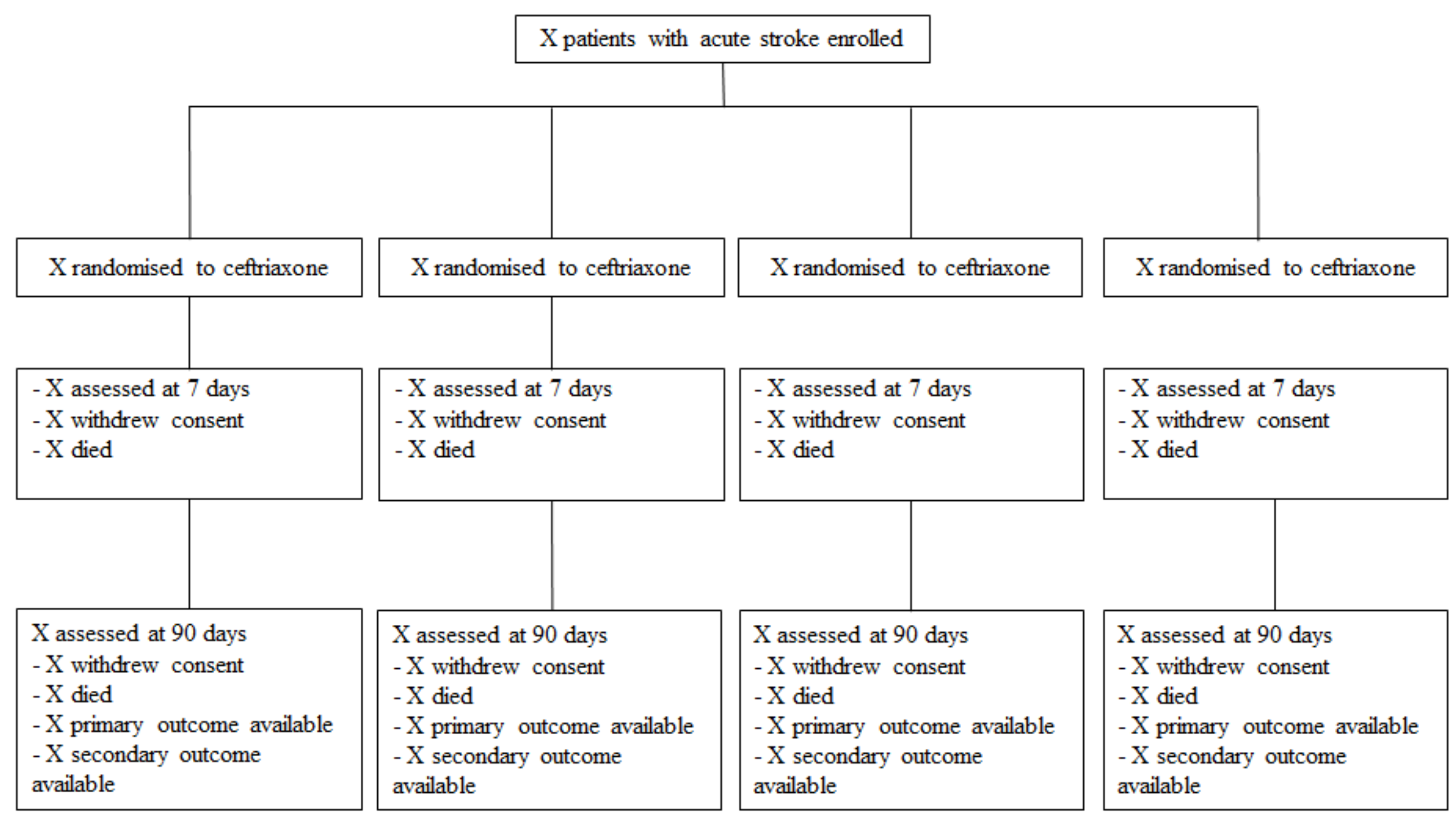


Figure 2 a/b/c. Distribution of modified Rankin Scale for each intervention using median mRS value for each participant

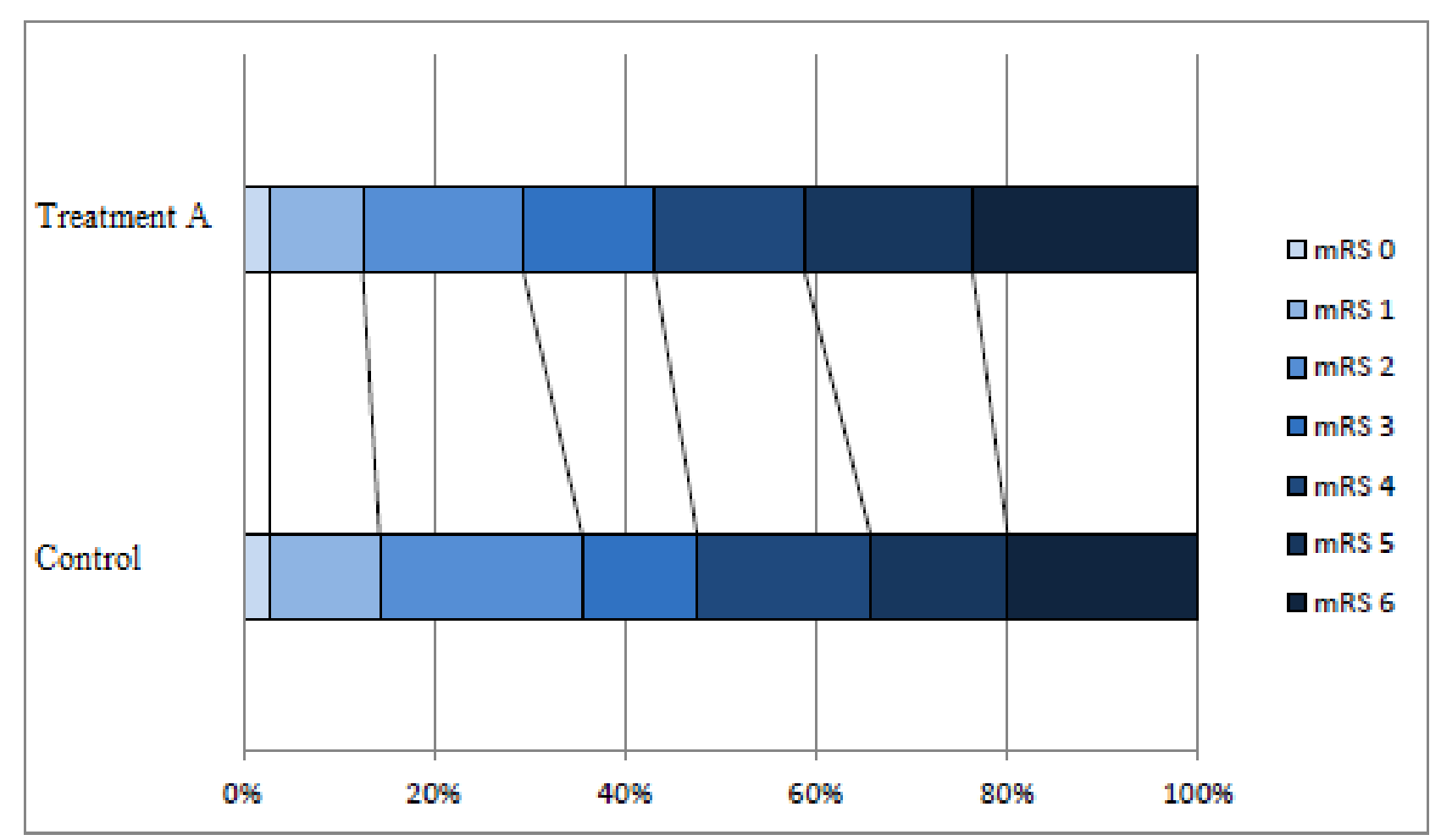

Example of a distribution of the modified Rankin Score at 3 months. The figure is an example, with dummy treatments and scores. 
Figure 3. Subgroup analysis - shown as forest plot. Adjusted analysis with interaction term

\begin{tabular}{|c|c|c|c|c|c|c|c|c|c|c|c|c|}
\hline & Paracetamol & Control & $\begin{array}{c}\mathrm{aOR} \\
(95 \% \\
\mathrm{CI})\end{array}$ & $\begin{array}{l}\text { Inter- } \\
\text { action } \\
\text { P }\end{array}$ & Metoclopramide & Control & $\begin{array}{c}\mathrm{aOR} \\
(95 \% \\
\mathrm{CI})\end{array}$ & $\begin{array}{c}\text { Inter- } \\
\text { action } \\
\text { P }\end{array}$ & Ceftriaxone & Control & $\begin{array}{c}\mathrm{aOR} \\
(95 \% \\
\mathrm{CI})\end{array}$ & $\begin{array}{c}\text { Inter- } \\
\text { action } \\
\text { P }\end{array}$ \\
\hline Age & & & & + & & & & + & & & & + \\
\hline Age $<75$ years & $\mathrm{n} / \mathrm{N}(\%)$ & $\mathrm{n} / \mathrm{N}(\%)$ & $\begin{array}{c}\mathrm{aOR} \\
(95 \% \mathrm{CI})\end{array}$ & & $\mathrm{n} / \mathrm{N}(\%)$ & $\mathrm{n} / \mathrm{N}(\%)$ & $\begin{array}{c}\mathrm{aOR} \\
(95 \% \mathrm{CI})\end{array}$ & & $\mathrm{n} / \mathrm{N}(\%)$ & $\mathrm{n} / \mathrm{N}(\%)$ & $\begin{array}{c}\mathrm{aOR} \\
(95 \% \mathrm{CI})\end{array}$ & \\
\hline Age $>75$ years & $\mathrm{n} / \mathrm{N}(\%)$ & $\mathrm{n} / \mathrm{N}(\%)$ & $\begin{array}{c}\mathrm{aOR} \\
(95 \% \mathrm{CI})\end{array}$ & & $\mathrm{n} / \mathrm{N}(\%)$ & $\mathrm{n} / \mathrm{N}(\%)$ & $\begin{array}{c}\mathrm{aOR} \\
(95 \% \mathrm{CI})\end{array}$ & & $\mathrm{n} / \mathrm{N}(\%)$ & $\mathrm{n} / \mathrm{N}(\%)$ & $\begin{array}{c}\mathrm{aOR} \\
(95 \% \mathrm{CI})\end{array}$ & \\
\hline Sex & & & & + & & & & + & & & & + \\
\hline Male & $\mathrm{n} / \mathrm{N}(\%)$ & $\mathrm{n} / \mathrm{N}(\%)$ & $\begin{array}{c}\mathrm{aOR} \\
(95 \% \mathrm{CI})\end{array}$ & & $\mathrm{n} / \mathrm{N}(\%)$ & $\mathrm{n} / \mathrm{N}(\%)$ & $\begin{array}{c}\mathrm{aOR} \\
(95 \% \mathrm{CI})\end{array}$ & & $\mathrm{n} / \mathrm{N}(\%)$ & $\mathrm{n} / \mathrm{N}(\%)$ & $\begin{array}{c}\mathrm{aOR} \\
(95 \% \mathrm{CI})\end{array}$ & \\
\hline Female & $\mathrm{n} / \mathrm{N}(\%)$ & $\mathrm{n} / \mathrm{N}(\%)$ & $\begin{array}{c}\mathrm{aOR} \\
(95 \% \mathrm{CI})\end{array}$ & & $\mathrm{n} / \mathrm{N}(\%)$ & $\mathrm{n} / \mathrm{N}(\%)$ & $\begin{array}{c}\mathrm{aOR} \\
(95 \% \mathrm{CI})\end{array}$ & & $\mathrm{n} / \mathrm{N}(\%)$ & $\mathrm{n} / \mathrm{N}(\%)$ & $\begin{array}{c}\mathrm{aOR} \\
(95 \% \mathrm{CI})\end{array}$ & \\
\hline Stroke type & & & & + & & & & + & & & & + \\
\hline Ischemic stroke & $\mathrm{n} / \mathrm{N}(\%)$ & $\mathrm{n} / \mathrm{N}(\%)$ & $\begin{array}{c}\mathrm{aOR} \\
(95 \% \mathrm{CI})\end{array}$ & & $\mathrm{n} / \mathrm{N}(\%)$ & $\mathrm{n} / \mathrm{N}(\%)$ & $\begin{array}{c}\mathrm{aOR} \\
(95 \% \mathrm{CI})\end{array}$ & & $\mathrm{n} / \mathrm{N}(\%)$ & $\mathrm{n} / \mathrm{N}(\%)$ & $\begin{array}{c}\mathrm{aOR} \\
(95 \% \mathrm{CI})\end{array}$ & \\
\hline $\begin{array}{l}\text { Intracerebral } \\
\text { haemorrhage }\end{array}$ & $\mathrm{n} / \mathrm{N}(\%)$ & $\mathrm{n} / \mathrm{N}(\%)$ & $\begin{array}{c}\mathrm{aOR} \\
(95 \% \mathrm{CI})\end{array}$ & & $\mathrm{n} / \mathrm{N}(\%)$ & $\mathrm{n} / \mathrm{N}(\%)$ & $\begin{array}{c}\mathrm{aOR} \\
(95 \% \mathrm{CI})\end{array}$ & & $\mathrm{n} / \mathrm{N}(\%)$ & $\mathrm{n} / \mathrm{N}(\%)$ & $\begin{array}{c}\mathrm{aOR} \\
(95 \% \mathrm{CI})\end{array}$ & \\
\hline Other diagnosis & $\mathrm{n} / \mathrm{N}(\%)$ & $\mathrm{n} / \mathrm{N}(\%)$ & $\begin{array}{c}\mathrm{aOR} \\
(95 \% \mathrm{CI})\end{array}$ & & $\mathrm{n} / \mathrm{N}(\%)$ & $\mathrm{n} / \mathrm{N}(\%)$ & $\begin{array}{c}\mathrm{aOR} \\
(95 \% \mathrm{CI})\end{array}$ & & $\mathrm{n} / \mathrm{N}(\%)$ & $\mathrm{n} / \mathrm{N}(\%)$ & $\begin{array}{c}\mathrm{aOR} \\
(95 \% \mathrm{CI})\end{array}$ & \\
\hline Stroke severity & & & & + & & & & + & & & & + \\
\hline NIHSS 6-12 & $\mathrm{n} / \mathrm{N}(\%)$ & $\mathrm{n} / \mathrm{N}(\%)$ & $\begin{array}{c}\mathrm{aOR} \\
(95 \% \mathrm{CI})\end{array}$ & & $\mathrm{n} / \mathrm{N}(\%)$ & $\mathrm{n} / \mathrm{N}(\%)$ & $\begin{array}{c}\mathrm{aOR} \\
(95 \% \mathrm{CI})\end{array}$ & & $\mathrm{n} / \mathrm{N}(\%)$ & $\mathrm{n} / \mathrm{N}(\%)$ & $\begin{array}{c}\mathrm{aOR} \\
(95 \% \mathrm{CI})\end{array}$ & \\
\hline
\end{tabular}




\begin{tabular}{|c|c|c|c|c|c|c|c|c|}
\hline NIHSS > 12 & $\mathrm{n} / \mathrm{N}(\%)$ & $\mathrm{n} / \mathrm{N}(\%)$ & $\begin{array}{c}\mathrm{aOR} \\
(95 \% \mathrm{CI})\end{array}$ & $\mathrm{n} / \mathrm{N}(\%)$ & $\mathrm{n} / \mathrm{N}(\%)$ & $\begin{array}{c}\mathrm{aOR} \\
(95 \% \mathrm{CI})\end{array}$ & $\mathrm{n} / \mathrm{N}(\%)$ & $\begin{array}{c}\mathrm{aOR} \\
(95 \% \mathrm{CI})\end{array}$ \\
\hline $\begin{array}{l}\text { Diabetes } \\
\text { Mellitus }\end{array}$ & & & & & & & & \\
\hline Yes & $\mathrm{n} / \mathrm{N}(\%)$ & $\mathrm{n} / \mathrm{N}(\%)$ & $\begin{array}{c}\mathrm{aOR} \\
(95 \% \mathrm{CI})\end{array}$ & $\mathrm{n} / \mathrm{N}(\%)$ & $\mathrm{n} / \mathrm{N}(\%)$ & $\begin{array}{c}\mathrm{aOR} \\
(95 \% \mathrm{CI})\end{array}$ & $\mathrm{n} / \mathrm{N}(\%)$ & $\begin{array}{c}\mathrm{aOR} \\
(95 \% \mathrm{CI})\end{array}$ \\
\hline No & $\mathrm{n} / \mathrm{N}(\%)$ & $\mathrm{n} / \mathrm{N}(\%)$ & $\begin{array}{c}\mathrm{aOR} \\
(95 \% \mathrm{CI})\end{array}$ & $\mathrm{n} / \mathrm{N}(\%)$ & $\mathrm{n} / \mathrm{N}(\%)$ & $\begin{array}{c}\mathrm{aOR} \\
(95 \% \mathrm{CI})\end{array}$ & $\mathrm{n} / \mathrm{N}(\%)$ & $\begin{array}{c}\mathrm{aOR} \\
(95 \% \mathrm{CI})\end{array}$ \\
\hline $\begin{array}{l}\text { Atrial } \\
\text { Fibrillation }\end{array}$ & & & & & & & & \\
\hline Yes & $\mathrm{n} / \mathrm{N}(\%)$ & $\mathrm{n} / \mathrm{N}(\%)$ & $\begin{array}{c}\mathrm{aOR} \\
(95 \% \mathrm{CI})\end{array}$ & $\mathrm{n} / \mathrm{N}(\%)$ & $\mathrm{n} / \mathrm{N}(\%)$ & $\begin{array}{c}\mathrm{aOR} \\
(95 \% \mathrm{CI})\end{array}$ & $\mathrm{n} / \mathrm{N}(\%)$ & $\begin{array}{c}\mathrm{aOR} \\
(95 \% \mathrm{CI})\end{array}$ \\
\hline No & $\mathrm{n} / \mathrm{N}(\%)$ & $\mathrm{n} / \mathrm{N}(\%)$ & $\begin{array}{c}\mathrm{aOR} \\
(95 \% \mathrm{CI})\end{array}$ & $\mathrm{n} / \mathrm{N}(\%)$ & $\mathrm{n} / \mathrm{N}(\%)$ & $\begin{array}{c}\mathrm{aOR} \\
(95 \% \mathrm{CI})\end{array}$ & $\mathrm{n} / \mathrm{N}(\%)$ & $\begin{array}{c}\mathrm{aOR} \\
(95 \% \mathrm{CI})\end{array}$ \\
\hline $\begin{array}{l}\text { Pre-stroke } \\
\text { mRS }\end{array}$ & & & & & & & & \\
\hline 0 & $\mathrm{n} / \mathrm{N}(\%)$ & $\mathrm{n} / \mathrm{N}(\%)$ & $\begin{array}{c}\mathrm{aOR} \\
(95 \% \mathrm{CI})\end{array}$ & $\mathrm{n} / \mathrm{N}(\%)$ & $\mathrm{n} / \mathrm{N}(\%)$ & $\begin{array}{c}\mathrm{aOR} \\
(95 \% \mathrm{CI})\end{array}$ & $\mathrm{n} / \mathrm{N}(\%)$ & $\begin{array}{c}\mathrm{aOR} \\
(95 \% \mathrm{CI})\end{array}$ \\
\hline$>0$ & $\mathrm{n} / \mathrm{N}(\%)$ & $\mathrm{n} / \mathrm{N}(\%)$ & $\begin{array}{c}\mathrm{aOR} \\
(95 \% \mathrm{CI})\end{array}$ & $\mathrm{n} / \mathrm{N}(\%)$ & $\mathrm{n} / \mathrm{N}(\%)$ & $\begin{array}{c}\mathrm{aOR} \\
(95 \% \mathrm{CI})\end{array}$ & $\mathrm{n} / \mathrm{N}(\%)$ & $\begin{array}{c}\mathrm{aOR} \\
(95 \% \mathrm{CI})\end{array}$ \\
\hline $\begin{array}{l}\text { Treatment with } \\
\text { alteplase }\end{array}$ & & & & & & & & \\
\hline Yes & $\mathrm{n} / \mathrm{N}(\%)$ & $\mathrm{n} / \mathrm{N}(\%)$ & $\begin{array}{c}\mathrm{aOR} \\
(95 \% \mathrm{CI})\end{array}$ & $\mathrm{n} / \mathrm{N}(\%)$ & $\mathrm{n} / \mathrm{N}(\%)$ & $\begin{array}{c}\mathrm{aOR} \\
(95 \% \mathrm{CI})\end{array}$ & $\mathrm{n} / \mathrm{N}(\%)$ & $\begin{array}{c}\mathrm{aOR} \\
(95 \% \mathrm{CI})\end{array}$ \\
\hline No & $\mathrm{n} / \mathrm{N}(\%)$ & $\mathrm{n} / \mathrm{N}(\%)$ & $\begin{array}{c}\mathrm{aOR} \\
(95 \% \mathrm{CI})\end{array}$ & $\mathrm{n} / \mathrm{N}(\%)$ & $\mathrm{n} / \mathrm{N}(\%)$ & $\begin{array}{c}\mathrm{aOR} \\
(95 \% \mathrm{CI})\end{array}$ & $\mathrm{n} / \mathrm{N}(\%)$ & $\begin{array}{c}\mathrm{aOR} \\
(95 \% \mathrm{CI})\end{array}$ \\
\hline
\end{tabular}




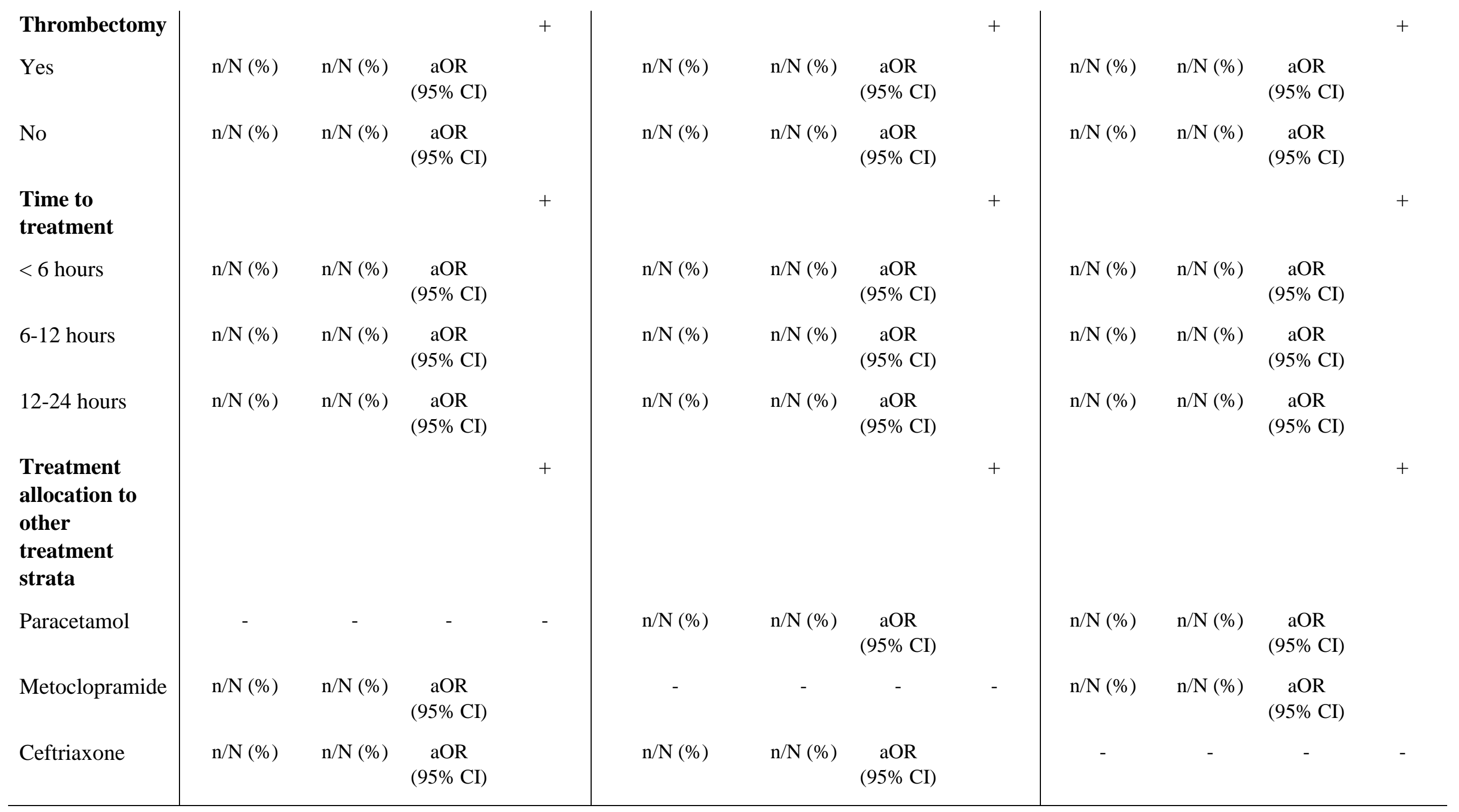

Data are $\mathrm{n} / \mathrm{N}(\%)$. aOR: adjusted odds ratio. Comparison by adjusted ordinal logistic regression with adjustment for an interaction term. This table will be presented as Forest plots in the final publication. 


\section{Supplement Table 1. Protocol violations in eligibility}

\begin{tabular}{|c|c|c|c|c|c|c|}
\hline & Paracetamol & Control & Metoclopramide & Control & Ceftriaxone & Control \\
\hline & $\mathrm{N}$ & $\mathrm{N}$ & $\mathrm{N}$ & $\mathrm{N}$ & $\mathrm{N}$ & $\mathrm{N}$ \\
\hline Other diagnosis than stroke & $\mathrm{n}(\%)$ & $\mathrm{n}(\%)$ & $\mathrm{n}(\%)$ & $\mathrm{n}(\%)$ & $\mathrm{n}(\%)$ & $\mathrm{n}(\%)$ \\
\hline NIHSS score of $\leq 5$ & $\mathrm{n}(\%)$ & $\mathrm{n}(\%)$ & $\mathrm{n}(\%)$ & $\mathrm{n}(\%)$ & $\mathrm{n}(\%)$ & $\mathrm{n}(\%)$ \\
\hline Age $\leq 65$ years & $\mathrm{n}(\%)$ & $\mathrm{n}(\%)$ & $\mathrm{n}(\%)$ & $\mathrm{n}(\%)$ & $\mathrm{n}(\%)$ & $\mathrm{n}(\%)$ \\
\hline Start treatment $>24$ hours & $\mathrm{n}(\%)$ & $\mathrm{n}(\%)$ & $\mathrm{n}(\%)$ & $\mathrm{n}(\%)$ & $\mathrm{n}(\%)$ & $\mathrm{n}(\%)$ \\
\hline $\begin{array}{l}\text { Inclusion with active infection } \\
\text { requiring antibiotic treatment }\end{array}$ & $\mathrm{n}(\%)$ & $\mathrm{n}(\%)$ & $\mathrm{n}(\%)$ & $\mathrm{n}(\%)$ & $\mathrm{n}(\%)$ & $\mathrm{n}(\%)$ \\
\hline Pre-stroke $\mathrm{mRS} \geq 4$ & $\mathrm{n}(\%)$ & $\mathrm{n}(\%)$ & $\mathrm{n}(\%)$ & $\mathrm{n}(\%)$ & $\mathrm{n}(\%)$ & $\mathrm{n}(\%)$ \\
\hline Death is imminent & $\mathrm{n}(\%)$ & $\mathrm{n}(\%)$ & $\mathrm{n}(\%)$ & $\mathrm{n}(\%)$ & $\mathrm{n}(\%)$ & $\mathrm{n}(\%)$ \\
\hline $\begin{array}{l}\text { Inclusion in treatment arm despite } \\
\text { contra-indication }\end{array}$ & $\mathrm{n}(\%)$ & $\mathrm{n}(\%)$ & $\mathrm{n}(\%)$ & $\mathrm{n}(\%)$ & $\mathrm{n}(\%)$ & $\mathrm{n}(\%)$ \\
\hline
\end{tabular}

Data are n (\%). mRS, modified Rankin Scale. 
Supplement Table 2. Compliance and cross-over in first 7 days

\begin{tabular}{|c|c|c|c|c|c|c|c|c|c|}
\hline & Paracetamol & Control & $\mathrm{P}$ & Metoclopramide & Control & $\mathrm{P}$ & Ceftriaxone & Control & $\mathrm{P}$ \\
\hline & $\mathrm{N}$ & $\mathrm{N}$ & & $\mathrm{N}$ & $\mathrm{N}$ & & $\mathrm{N}$ & $\mathrm{N}$ & \\
\hline $\begin{array}{l}\text { Received all allocated } \\
\text { dosages }\end{array}$ & $\mathrm{n}(\%)$ & - & - & $\mathrm{n}(\%)$ & - & - & $\mathrm{n}(\%)$ & - & - \\
\hline $\begin{array}{l}\text { Received } 75-99 \% \text { of } \\
\text { dosages }\end{array}$ & $\mathrm{n}(\%)$ & - & - & $\mathrm{n}(\%)$ & - & - & $\mathrm{n}(\%)$ & - & - \\
\hline $\begin{array}{l}\text { Received } 50-<75 \% \text { of } \\
\text { dosages }\end{array}$ & $\mathrm{n}(\%)$ & - & - & $\mathrm{n}(\%)$ & - & - & $\mathrm{n}(\%)$ & - & - \\
\hline $\begin{array}{l}\text { Received } 25-<50 \% \text { of } \\
\text { dosages }\end{array}$ & $\mathrm{n}(\%)$ & - & - & $\mathrm{n}(\%)$ & - & - & $\mathrm{n}(\%)$ & - & - \\
\hline $\begin{array}{l}\text { Received } 0-<25 \% \text { of } \\
\text { dosages }\end{array}$ & $\mathrm{n}(\%)$ & - & - & $\mathrm{n}(\%)$ & - & - & $\mathrm{n}(\%)$ & - & - \\
\hline $\begin{array}{l}\text { Received any antibiotic } \\
\text { drug }\end{array}$ & $\mathrm{n}(\%)$ & $\mathrm{n}(\%)$ & & $\mathrm{n}(\%)$ & $\mathrm{n}(\%)$ & & $\mathrm{n}(\%)$ & $\mathrm{n}(\%)$ & \\
\hline $\begin{array}{l}\text { Received any } \\
\text { antipyretic drug }\end{array}$ & $\mathrm{n}(\%)$ & $\mathrm{n}(\%)$ & & $\mathrm{n}(\%)$ & $\mathrm{n}(\%)$ & & $\mathrm{n}(\%)$ & $\mathrm{n}(\%)$ & \\
\hline $\begin{array}{l}\text { Received any } \\
\text { antipyretic drug for four } \\
\text { days at least once }\end{array}$ & $\mathrm{n}(\%)$ & $\mathrm{n}(\%)$ & & $\mathrm{n}(\%)$ & $\mathrm{n}(\%)$ & & $\mathrm{n}(\%)$ & $\mathrm{n}(\%)$ & \\
\hline $\begin{array}{l}\text { Received any anti- } \\
\text { emetic drug }\end{array}$ & $\mathrm{n}(\%)$ & $\mathrm{n}(\%)$ & & $\mathrm{n}(\%)$ & $\mathrm{n}(\%)$ & & $\mathrm{n}(\%)$ & $\mathrm{n}(\%)$ & \\
\hline
\end{tabular}




\begin{tabular}{l|l|llll}
$\begin{array}{l}\text { Received any anti- } \\
\text { emetic drug for four } \\
\text { days at least once }\end{array}$ & $\mathrm{n}(\%)$ & $\mathrm{n}(\%)$ & $\mathrm{n}(\%)$ & $\mathrm{n}(\%)$ & $\mathrm{n}(\%)$ \\
\hline
\end{tabular}

Data are $\mathrm{n}(\%)$. Comparisons made by binary logistic regression. 
Supplement Table 3. Secondary outcomes and treatment restrictions at 7 days

\begin{tabular}{|c|c|c|c|c|c|c|c|c|c|c|}
\hline & Analysis & Paracetamol & Control & $\begin{array}{c}\text { OR } \\
(95 \% \\
\mathrm{CI})\end{array}$ & Metoclopramide & Control & $\begin{array}{c}\text { OR } \\
(95 \% \\
\text { CI })\end{array}$ & Ceftriaxone & Control & $\begin{array}{c}\text { OR } \\
(95 \% \\
\text { CI })\end{array}$ \\
\hline \multicolumn{11}{|l|}{ mRS, median } \\
\hline All patients & aOLR & $\begin{array}{c}\text { Median } \\
{[\mathrm{IQR}]}\end{array}$ & $\begin{array}{c}\text { Median } \\
\text { [IQR] }\end{array}$ & $\begin{array}{c}\mathrm{aOR} \\
(95 \% \\
\mathrm{CI})\end{array}$ & Median [IQR] & $\begin{array}{c}\text { Median } \\
\text { [IQR] }\end{array}$ & $\begin{array}{c}\mathrm{aOR} \\
(95 \% \\
\mathrm{CI})\end{array}$ & $\begin{array}{c}\text { Median } \\
{[\mathrm{IQR}]}\end{array}$ & $\begin{array}{c}\text { Median } \\
\text { [IQR] }\end{array}$ & $\begin{array}{c}\mathrm{aOR} \\
(95 \% \\
\mathrm{CI})\end{array}$ \\
\hline Ischemic stroke & aOLR & $\begin{array}{c}\text { Median } \\
{[\mathrm{IQR}]}\end{array}$ & $\begin{array}{c}\text { Median } \\
\text { [IQR }]\end{array}$ & $\begin{array}{c}\mathrm{aOR} \\
(95 \% \\
\mathrm{CI})\end{array}$ & Median [IQR] & $\begin{array}{c}\text { Median } \\
\text { [IQR] }\end{array}$ & $\begin{array}{c}\mathrm{aOR} \\
(95 \% \\
\mathrm{CI})\end{array}$ & $\begin{array}{c}\text { Median } \\
\text { [IQR] }\end{array}$ & $\begin{array}{c}\text { Median } \\
{[\mathrm{IQR}]}\end{array}$ & $\begin{array}{c}\mathrm{aOR} \\
(95 \% \\
\mathrm{CI})\end{array}$ \\
\hline Haemorrhagic stroke & aOLR & $\begin{array}{c}\text { Median } \\
{[\mathrm{IQR}]}\end{array}$ & $\begin{array}{c}\text { Median } \\
{[\mathrm{IQR}]}\end{array}$ & $\begin{array}{c}\mathrm{aOR} \\
(95 \% \\
\mathrm{CI})\end{array}$ & Median [IQR] & $\begin{array}{c}\text { Median } \\
\text { [IQR] }\end{array}$ & $\begin{array}{c}\mathrm{aOR} \\
(95 \% \\
\mathrm{CI})\end{array}$ & $\begin{array}{c}\text { Median } \\
{[\mathrm{IQR}]}\end{array}$ & $\begin{array}{c}\text { Median } \\
{[\mathrm{IQR}]}\end{array}$ & $\begin{array}{c}\mathrm{aOR} \\
(95 \% \\
\mathrm{CI})\end{array}$ \\
\hline Mortality at 7 days & aBLR & $\mathrm{n}(\%)$ & $\mathrm{n}(\%)$ & $\begin{array}{c}\mathrm{aOR} \\
(95 \% \mathrm{CI})\end{array}$ & $\mathrm{n}(\%)$ & $\mathrm{n}(\%)$ & $\begin{array}{c}\mathrm{aOR} \\
(95 \% \mathrm{CI})\end{array}$ & $\mathrm{n}(\%)$ & $\mathrm{n}(\%)$ & $\begin{array}{c}\mathrm{aOR} \\
(95 \% \mathrm{CI})\end{array}$ \\
\hline $\begin{array}{l}\text { Any treatment } \\
\text { restriction }\end{array}$ & - & $\mathrm{n}(\%)$ & $\mathrm{n}(\%)$ & - & $\mathrm{n}(\%)$ & $\mathrm{n}(\%)$ & - & $\mathrm{n}(\%)$ & $\mathrm{n}(\%)$ & - \\
\hline \multicolumn{11}{|l|}{ Infection } \\
\hline All infections & aBLR & $\mathrm{n}(\%)$ & $\mathrm{n}(\%)$ & $\begin{array}{c}\mathrm{aOR} \\
(95 \% \\
\mathrm{CI})\end{array}$ & $\mathrm{n}(\%)$ & $\mathrm{n}(\%)$ & $\begin{array}{c}\mathrm{aOR} \\
(95 \% \\
\mathrm{CI})\end{array}$ & $\mathrm{n}(\%)$ & $\mathrm{n}(\%)$ & $\begin{array}{c}\mathrm{aOR} \\
(95 \% \\
\mathrm{CI})\end{array}$ \\
\hline Pneumonia & aBLR & $\mathrm{n}(\%)$ & $\mathrm{n}(\%)$ & $\begin{array}{c}\mathrm{aOR} \\
(95 \% \\
\mathrm{CI})\end{array}$ & $\mathrm{n}(\%)$ & $\mathrm{n}(\%)$ & $\begin{array}{c}\mathrm{aOR} \\
(95 \% \\
\mathrm{CI})\end{array}$ & $\mathrm{n}(\%)$ & $\mathrm{n}(\%)$ & $\begin{array}{c}\mathrm{aOR} \\
(95 \% \\
\mathrm{CI})\end{array}$ \\
\hline
\end{tabular}




\begin{tabular}{|c|c|c|c|c|c|c|c|c|c|c|}
\hline Urinary tract infection & aBLR & $\mathrm{n}(\%)$ & $\mathrm{n}(\%)$ & $\begin{array}{c}\mathrm{aOR} \\
(95 \% \\
\mathrm{CI})\end{array}$ & $\mathrm{n}(\%)$ & $\mathrm{n}(\%)$ & $\begin{array}{c}\mathrm{aOR} \\
(95 \% \\
\mathrm{CI})\end{array}$ & $\mathrm{n}(\%)$ & $\mathrm{n}(\%)$ & $\begin{array}{c}\mathrm{aOR} \\
(95 \% \\
\mathrm{CI})\end{array}$ \\
\hline Other infections & aBLR & $\mathrm{n}(\%)$ & $\mathrm{n}(\%)$ & $\begin{array}{c}\mathrm{aOR} \\
(95 \% \\
\mathrm{CI})\end{array}$ & $\mathrm{n}(\%)$ & $\mathrm{n}(\%)$ & $\begin{array}{c}\mathrm{aOR} \\
(95 \% \\
\mathrm{CI})\end{array}$ & $\mathrm{n}(\%)$ & $\mathrm{n}(\%)$ & $\begin{array}{c}\mathrm{aOR} \\
(95 \% \\
\mathrm{CI})\end{array}$ \\
\hline \multicolumn{11}{|l|}{$\begin{array}{l}\text { Infections based on } \\
\text { expert panel }\end{array}$} \\
\hline All infections & aBLR & $\mathrm{n}(\%)$ & $\mathrm{n}(\%)$ & $\begin{array}{c}\mathrm{aOR} \\
(95 \% \\
\mathrm{CI})\end{array}$ & $\mathrm{n}(\%)$ & $\mathrm{n}(\%)$ & $\begin{array}{c}\mathrm{aOR} \\
(95 \% \\
\mathrm{CI})\end{array}$ & $\mathrm{n}(\%)$ & $\mathrm{n}(\%)$ & $\begin{array}{c}\mathrm{aOR} \\
(95 \% \\
\mathrm{CI})\end{array}$ \\
\hline Pneumonia & aBLR & $\mathrm{n}(\%)$ & $\mathrm{n}(\%)$ & $\begin{array}{c}\mathrm{aOR} \\
(95 \% \\
\mathrm{CI})\end{array}$ & n $(\%)$ & $\mathrm{n}(\%)$ & $\begin{array}{c}\mathrm{aOR} \\
(95 \% \\
\mathrm{CI})\end{array}$ & $\mathrm{n}(\%)$ & $\mathrm{n}(\%)$ & $\begin{array}{c}\mathrm{aOR} \\
(95 \% \\
\mathrm{CI})\end{array}$ \\
\hline Urinary tract infection & aBLR & $\mathrm{n}(\%)$ & $\mathrm{n}(\%)$ & $\begin{array}{c}\mathrm{aOR} \\
(95 \% \\
\mathrm{CI})\end{array}$ & $\mathrm{n}(\%)$ & $\mathrm{n}(\%)$ & $\begin{array}{c}\mathrm{aOR} \\
(95 \% \\
\mathrm{CI})\end{array}$ & $\mathrm{n}(\%)$ & $\mathrm{n}(\%)$ & $\begin{array}{c}\mathrm{aOR} \\
(95 \% \\
\mathrm{CI})\end{array}$ \\
\hline Other infections & aBLR & $\mathrm{n}(\%)$ & $\mathrm{n}(\%)$ & $\begin{array}{c}\mathrm{aOR} \\
(95 \% \\
\mathrm{CI})\end{array}$ & $\mathrm{n}(\%)$ & $\mathrm{n}(\%)$ & $\begin{array}{c}\mathrm{aOR} \\
(95 \% \\
\mathrm{CI})\end{array}$ & $\mathrm{n}(\%)$ & $\mathrm{n}(\%)$ & $\begin{array}{c}\mathrm{aOR} \\
(95 \% \\
\mathrm{CI})\end{array}$ \\
\hline $\begin{array}{l}\text { Antimicrobial use and } \\
\text { resistance } \\
\text { 3rd generation } \\
\text { cephalosporin resistance }\end{array}$ & aBLR & $\mathrm{n}(\%)$ & $\mathrm{n}(\%)$ & $\begin{array}{c}\mathrm{aOR} \\
(95 \% \\
\mathrm{CI})\end{array}$ & $\mathrm{n}(\%)$ & $\mathrm{n}(\%)$ & $\begin{array}{c}\mathrm{aOR} \\
(95 \% \\
\mathrm{CI})\end{array}$ & $\mathrm{n}(\%)$ & $\mathrm{n}(\%)$ & $\begin{array}{c}\mathrm{aOR} \\
(95 \% \\
\mathrm{CI})\end{array}$ \\
\hline $\begin{array}{l}\text { Antimicrobial use } \\
\text { during first } 7 \text { days* }\end{array}$ & & DDD & DDD & - & DDD & DDD & - & DDD & DDD & - \\
\hline
\end{tabular}


mRS, modified Rankin Scale. Data are n (\%) or median [IQR]. aOR: adjusted odds ratio. Comparison by adjusted ordinal logistic regression (aOLR) or binary logistic regression (aBLR).

* Converted to units of defined daily doses according to the classification of the WHO Anatomical Therapeutic Chemical Classification System with Defined Daily Doses (DDD) Index; 
Supplement Table 4. Overview of safety

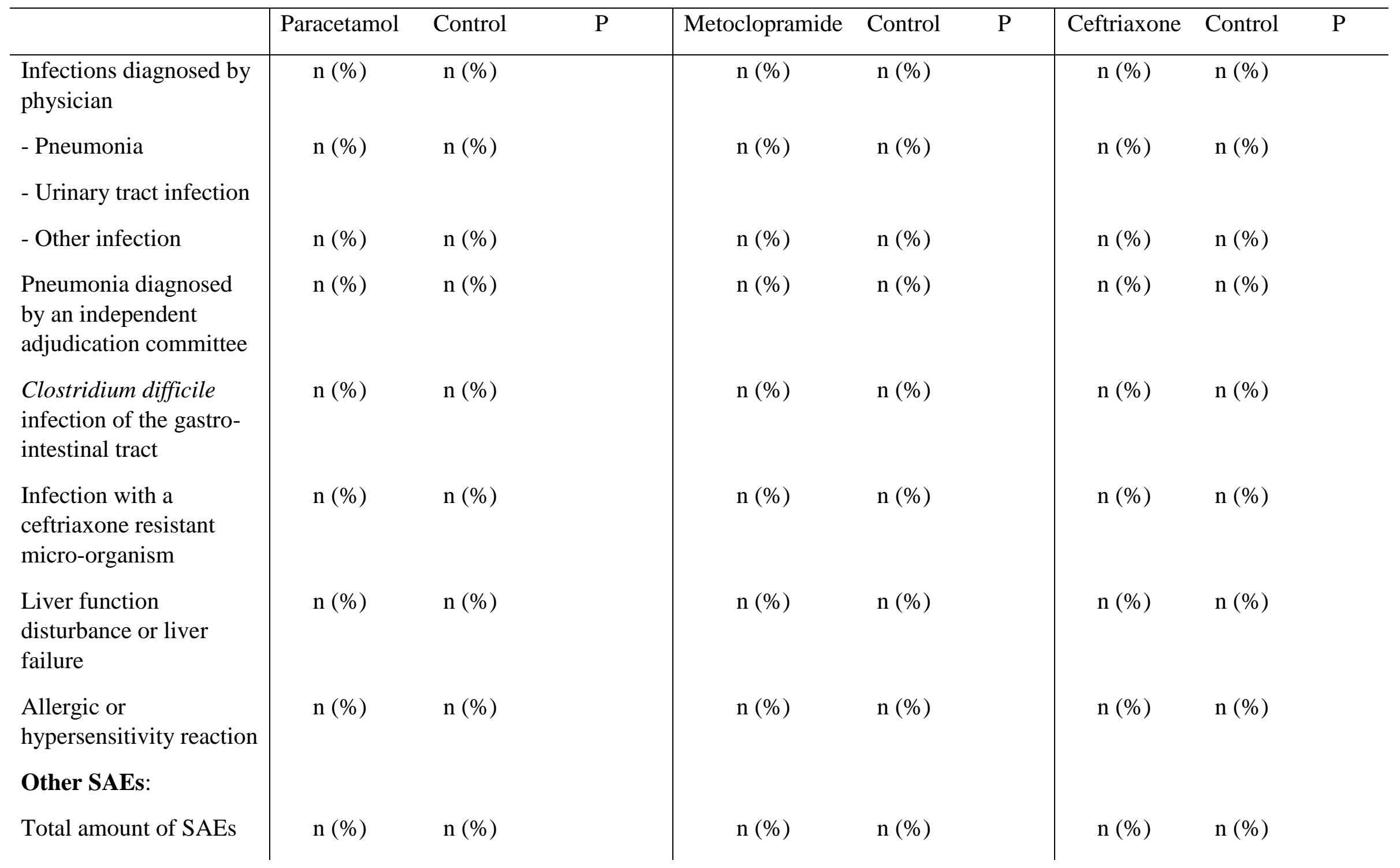




\begin{tabular}{l|cc|cc|cc}
$\begin{array}{l}\text { Total amount of related } \\
\text { SAEs (SARs or }\end{array}$ & $\mathrm{n}(\%)$ & $\mathrm{n}(\%)$ & $\mathrm{n}(\%)$ & $\mathrm{n}(\%)$ & $\mathrm{n}(\%)$ & $\mathrm{n}(\%)$ \\
$\begin{array}{l}\text { SUSARs) } \\
\begin{array}{l}\text { Total amount of } \\
\text { SUSARs }\end{array}\end{array}$ & $\mathrm{n}(\%)$ & $\mathrm{n}(\%)$ & $\mathrm{n}(\%)$ & $\mathrm{n}(\%)$ & $\mathrm{n}(\%)$ & $\mathrm{n}(\%)$ \\
\hline
\end{tabular}

Data are n (\%). SAE, Severe Adverse Event; SAR, Severe Adverse Reaction; SUSAR, Severe Unexpected Serious Adverse Reaction. Comparisons made by binary logistic regression. 
Supplement Figure 1 a/b/c. Kaplan Meier of death for each intervention 


\section{Declarations}

\section{Ethics approval and consent to participate}

The primary Ethics approval for the PRECIOUS trial has been provided by the Medical Ethics Committee of the University Medical Center Utrecht, Utrecht, the Netherlands (NL54304.041.13). We have obtained informed consent from all participants in the study

Consent for publication: Not applicable.

\section{Availability of data and materials}

The details of the study protocol have been published earlier (20). After publication of the trial, to promote the independent re-use of PRECIOUS data, a coded dataset will be made available in a public data repository within 18 months of the final follow-up of the last patient. Coded data will also be included in VISTA.

\section{Competing interests}

HBvdW served as a consultant to Boehringer Ingelheim, Bayer and LivaNova. PMB has served on advisory boards with DiaMedica, Moleac, Nestle, Phagenesis, Platelet Solutions and Sanofi

\section{Funding}

PRECIOUS is funded by the European Union's Horizon, 2020 research and innovation programme (grant no. 634809).

\section{Authors' contributions}

HBvdW is the PRECIOUS coordinating investigator. All authors contributed to the design of the statistical analysis. JdJ wrote the first draft of the manuscript and all author authors reviewed the manuscript carefully. All authors read and approved the final version of the manuscript. 


\section{List of abbreviations}

BI

BLR

BP

CCMO

CONSORT

CPHR

DIM

ESBL

EQ-5D-5L

EQ-VAS

GCP

DDD

DSMB

HR

$\mathrm{ICH}$

IMP

IQR

MD

MLR

MoCA

mRS

NIHSS

NSTU

OLR

OR
Barthel Index

Binary logistic regression

Blood Pressure

Centrale Commissie Mensgebonden Onderzoek

Consolidated Standards of Reporting Trials

Cox proportional hazards regression

Difference in means

Extended-Spectrum Beta-Lactamase

EuroQol 5D-5L

EQ-visual analogue scale

Good Clinical Practice

Daily Defined Dose

Data and Safety Monitoring Board

Hazard Ratio

International Conference on Harmonisation

Investigational Medicinal Product

Interquartile Range

Medical Doctor

Multiple linear regression

Montreal Cognitive Assessment

Modified Rankin Scale

National Institutes of Health Stroke Scale

Nottingham Stroke Trial Unit

Ordinal logistic regression

Odds ratio 
$\mathrm{PhD}$

PCR

PRECIOUS

PROBE

SAE

SAP

SAR

SD

SUSAR

UMC

UNOTT

VISTA

WHO
Doctor of Philosophy

Polymerase chain reaction

PREvention of Complications to Improve OUtcome in elderly

patients with acute Stroke

Prospective randomised open blinded end-point

Serious Adverse Event

Statistical Analysis Plan

Serious Adverse Reaction

Standard Deviation

Suspected Unexpected Serious Adverse Reaction

University Medical Center

University of Nottingham

Virtual International Stroke Trials Archive

World Health Organisation 


\section{Supplementary appendix}

List of PRECIOUS partners

\begin{tabular}{|c|c|}
\hline Affiliation & Investigator(s) \\
\hline $\begin{array}{l}\text { Department of Neurology and Neurosurgery, } \\
\text { Brain Center, University Medical Center } \\
\text { Utrecht, Utrecht University, Utrecht, The } \\
\text { Netherlands. }\end{array}$ & $\begin{array}{l}\text { Hendrik Reinink, Jeroen C de Jonge, H Bart } \\
\text { van der Worp }\end{array}$ \\
\hline $\begin{array}{l}\text { Stroke Trials Unit, Division of Clinical } \\
\text { Neuroscience, University of Nottingham, } \\
\text { Nottingham, United Kingdom }\end{array}$ & Philip M Bath \\
\hline $\begin{array}{l}\text { Department of Neurology, Academic Medical } \\
\text { Center, Amsterdam Neuroscience, Amsterdam, } \\
\text { The Netherlands }\end{array}$ & Diederik van de Beek \\
\hline $\begin{array}{l}\text { Department of Internal Medicine, Oslo, } \\
\text { University Hospital, Oslo, Norway }\end{array}$ & Eivind Berge \\
\hline $\begin{array}{l}\text { Department of Neurology and Stroke Unit, } \\
\text { ASST di Mantova, Mantua, Italy. }\end{array}$ & Alfonso Ciccone \\
\hline $\begin{array}{l}\text { Department of Neurology, University of } \\
\text { Debrecen, Debrecen, Hungary }\end{array}$ & Laszlo Csiba \\
\hline $\begin{array}{l}\text { European Clinical Research Infrastructure } \\
\text { Network (ECRIN), Paris, France }\end{array}$ & Jacques Demotes \\
\hline $\begin{array}{l}\text { Department of Neurology, Erasmus MC, } \\
\text { University Medical Center, Rotterdam, The } \\
\text { Netherlands }\end{array}$ & Diederik W Dippel \\
\hline $\begin{array}{l}\text { Department of Neurology and Neurosurgery, } \\
\text { University of Tartu, Tartu, Estonia }\end{array}$ & Janika Korv \\
\hline
\end{tabular}




\begin{tabular}{|l|l|}
\hline $\begin{array}{l}2^{\text {nd }} \text { Department of Neurology, Institute of } \\
\text { Psychiatry and Neurology, Warsaw, Poland }\end{array}$ & Iwona Kurkowska-Jastrzebska \\
\hline $\begin{array}{l}\text { Institute of Cardiovascular and Medical } \\
\text { sciences, University of Glasgow, Glasgow, } \\
\text { United Kingdom }\end{array}$ & Kennedy R Lees \\
\hline $\begin{array}{l}\text { Division of Clinical Neurosciences, Centre for } \\
\text { Clinical Brain Sciences, University of } \\
\text { Edinburgh, Edinburgh, United Kingdom }\end{array}$ & Malcolm R Macleod \\
\hline $\begin{array}{l}\text { Department of Medicine, Larissa University } \\
\text { Hospital, University of Thessaly, Larissa, } \\
\text { Greece }\end{array}$ & George Ntaios \\
\hline $\begin{array}{l}\text { Stroke Alliance for Europe (SAFE), Brussels, } \\
\text { Belgium }\end{array}$ & Gary Randall \\
\hline $\begin{array}{l}\text { Department of Neurology, Center for Clinical } \\
\text { Neurosciences, University Medical Center } \\
\text { Hamburg-Eppendorf, Hamburg, Germany. }\end{array}$ & Götz Thomalla \\
\hline
\end{tabular}




\section{References}

1. Kumar S, Selim MH, Caplan LR. Medical complications after stroke. Lancet Neurol. 2010;9(1):105-18.

2. Hesse K, Fulton RL, Abdul-Rahim AH, Lees KR, VISTA Collaborators. Characteristic adverse events and their incidence among patients participating in acute ischemic stroke trials. Stroke. 2014;45(9):2677-82.

3. Westendorp, Willeke; Vermeij, Jan-Dirk; Hilkens, Nina A; Brouwers, Matthijs C; Algra Ale; van der Worp, H Bart; Dippel, Diederik WJ; van de Beek, Diederik; Nederkoorn PJ.

Development and internal validation of a prediction rule for post-stroke infection and poststroke pneumonia in acute stroke patients. Eur Stroke J. 2018;0(0):1-9.

4. Westendorp WF, Nederkoorn PJ, Vermeij J-D, Dijkgraaf MG, de Beek D van. Post-stroke infection: A systematic review and meta-analysis. BMC Neurol. 2011;11(1):110.

5. den Hertog H, van der Worp B, van Gemert M, Dippel D. Therapeutic hypothermia in acute ischemic stroke. Expert Rev Neurother. 2007;7(2):155-64.

6. Greer DM, Funk SE, Reaven NL, Ouzounelli M, Uman GC. Impact of Fever on Outcome in Patients With Stroke and Neurologic Injury: A Comprehensive Meta-Analysis. Stroke. 2008;39(11):3029-35.

7. Saini M, Saqqur M, Kamruzzaman A, Lees KR, Shuaib A, VISTA Investigators. Effect of hyperthermia on prognosis after acute ischemic stroke. Stroke. 2009;40(9):3051-9.

8. Martino R, Foley N, Bhogal S, Diamant N, Speechley M, Teasell R. Dysphagia After Stroke: Incidence, Diagnosis, and Pulmonary Complications. Stroke. 2005;36(12):2756-63.

9. Geurts M, Scheijmans FE V, van Seeters T, Biessels GJ, Kappelle LJ, Velthuis BK, et al. Temporal profile of body temperature in acute ischemic stroke: relation to infarct size and outcome. BMC Neurol. 2016;16(1):233.

10. Cohen DL, Roffe C, Beavan J, Blackett B, Fairfield CA, Hamdy S, et al. Post-stroke dysphagia: A review and design considerations for future trials. Int J Stroke. 2016;11(4):399-411.

11. L.J. W, P. S, S. H, D.G. S, D.L. C, C. R, et al. Route of Feeding as a Proxy for Dysphagia After Stroke and the Effect of Transdermal Glyceryl Trinitrate: Data from the Efficacy of Nitric Oxide in Stroke Randomised Controlled Trial. Transl Stroke Res. 2018;9(2):120-9. 1

12. den Hertog HM, van der Worp HB, van Gemert HMA, Algra A, Kappelle LJ, van Gijn J, et al. An early rise in body temperature is related to unfavorable outcome after stroke: data from the PAIS study. J Neurol. 2011;258(2):302-7.

13. Westendorp WF, Vermeij J-D, Vermeij F, Den Hertog HM, Dippel DW, van de Beek D, et al. Antibiotic therapy for preventing infections in patients with acute stroke. Cochrane Database Syst Rev. 2012: CD008530.

14. den Hertog HM, van der Worp HB, van Gemert HMA, Algra A, Kappelle LJ, van Gijn J, et al. The Paracetamol (Acetaminophen) In Stroke (PAIS) trial: a multicentre, randomised, placebo-controlled, phase III trial. Lancet Neurol. 2009;8(5):434-40.

15. Warusevitane A, Karunatilake D, Sim J, Lally F, Roffe C. Safety and effect of metoclopramide to prevent pneumonia in patients with stroke fed via nasogastric tubes trial. 
Stroke. 2015;46(2):454-60.

16. Westendorp WF, Vermeij J-D, Zock E, Hooijenga IJ, Kruyt ND, Bosboom HJLW, et al. The Preventive Antibiotics in Stroke Study (PASS): a pragmatic randomised open-label masked endpoint clinical trial. Lancet. 2015;385(9977):1519-26.

17. Kalra L, Irshad S, Hodsoll J, Simpson M, Gulliford M, Smithard D, et al. Prophylactic antibiotics after acute stroke for reducing pneumonia in patients with dysphagia (STROKEINF): a prospective, cluster-randomised, open-label, masked endpoint, controlled clinical trial. Lancet. 2015;386(10006):1835-44.

18. Steiner T, Al-Shahi Salman R, Beer R, Christensen H, Cordonnier C, Csiba L, et al. European Stroke Organisation (ESO) guidelines for the management of spontaneous intracerebral hemorrhage. Int J Stroke. 2014;9(7):840-55.

19. Ntaios G, Dziedzic T, Michel P, Papavasileiou V, Petersson J, Staykov D, et al. European Stroke Organisation (ESO) guidelines for the management of temperature in patients with acute ischemic stroke. Int J Stroke. 2015;10(6):941-9.

20. Gamble C, Krishan A, Stocken D, Lewis S, Juszczak E, Doré C, et al. Guidelines for the content of statistical analysis plans in clinical trials. JAMA - J Am Med Assoc. 2017;318(23):2337-43.

21. Reinink H, de Jonge JC, Bath PM, van de Beek D, Berge E, Borregaard S, et al. PRECIOUS: PREvention of Complications to Improve OUtcome in elderly patients with acute Stroke. Rationale and design of a randomised, open, phase III, clinical trial with blinded outcome assessment. Eur Stroke J. 2018;3(3):291-8.

22. Lees KR, Bath PMW, Schellinger PD, Kerr DM, Fulton R, Hacke W, et al. Contemporary Outcome Measures in Acute Stroke Research. Stroke. 2012;43(4):1163-70.

23. McArthur KS, Johnson PCD, Quinn TJ, Higgins P, Langhorne P, Walters MR, et al. Improving the Efficiency of Stroke Trials. Stroke. 2013;44(12):3422-8.

24. Bath PMW, Lees KR, Schellinger PD, Altman H, Bland M, Hogg C, et al. Statistical analysis of the primary outcome in acute stroke trials. Stroke. 2012;43(4):1171-8. 